\title{
Daphnia Halloween genes that encode cytochrome P450s mediating the synthesis of the arthropod molting hormone: Evolutionary implications Kim F Rewitz ${ }^{1}$ and Lawrence I Gilbert*2
}

Address: ${ }^{1}$ Department of Science, Systems and Models, Roskilde University, Postbox 260, DK-4000 Roskilde, Denmark and ${ }^{2}$ Department of Biology, University of North Carolina, Chapel Hill, NC 27599-3280 USA

Email: Kim F Rewitz - rewitz@ruc.dk; Lawrence I Gilbert* - lgilbert@unc.edu

* Corresponding author

Published: 25 February 2008

BMC Evolutionary Biology 2008, 8:60 doi:10.1 186/I47|-2/48-8-60

This article is available from: http://www.biomedcentral.com/I47I-2/48/8/60

(c) 2008 Rewitz and Gilbert; licensee BioMed Central Ltd.

This is an Open Access article distributed under the terms of the Creative Commons Attribution License (http://creativecommons.org/licenses/by/2.0), which permits unrestricted use, distribution, and reproduction in any medium, provided the original work is properly cited.
Received: 15 November 2007

Accepted: 25 February 2008

\begin{abstract}
Background: In crustaceans and insects, development and reproduction are controlled by the steroid hormone, 20-hydroxyecdysone (20E). Like other steroids, 20E, is synthesized from cholesterol through reactions involving cytochrome P450s (CYPs). In insects, the CYP enzymes mediating 20E biosynthesis have been identified, but evidence of their probable presence in crustaceans is indirect, relying solely on the ability of crustaceans to synthesize $20 \mathrm{E}$.
\end{abstract}

Results: To investigate the presence of these genes in crustaceans, the genome of Daphnia pulex was examined for orthologs of these genes, the Halloween genes, encoding those biosynthetic CYP enzymes. Single homologs of spook-CYP307AI, phantom-CYP306AI, disembodied-CYP302AI, shadowCYP3 I5AI and shade-CYP3/4AI were identified in the Daphnia data base. Phylogenetic analysis indicates an orthologous relationship between the insect and Daphnia genes. Conserved intron/ exon structures and microsynteny further support the conclusion that these steroidogenic CYPs have been conserved in insects and crustaceans through some 400 million years of evolution.

Conclusion: Although these arthropod steroidogenic CYPs are related to steroidogenic CYPs in Caenorhabditis elegans and vertebrates, the data suggest that the arthropod steroidogenic CYPs became functionally specialized in a common ancestor of arthropods and are unique to these animals.

\section{Background}

Steroid hormones, regulate essential processes during development and reproduction, and are synthesized from cholesterol under the control of steroidogenic enzymes in the cytochrome P450 (CYP) family [1]. In Caenorhabditis elegans, insects and vertebrates, different steroids are produced to control developmental processes, suggesting that steroidogenic CYPs evolved and became functionally specialized in different lineages during evolution. In insects, a specific biosynthetic pathway yielding 20-hydroxyecdysone (20E), the arthropod molting hormone, evolved, whereas in the line leading to vertebrates, biosynthetic CYPs that produce the vertebrate-type steroids evolved [2]. Since there is some evidence of the presence of vertebrate-type sex steroids in invertebrates such as echinoderms and mollusks, although no unequivocal evidence that they can synthesize these steroids [3], the possibility remains that CYPs with the capacity to produce vertebrate- 
type sex steroids were present in the common ancestor even before the protostome-deuterostome split. Thus, the evolution of steroidogenic CYPs is still an open question.

Crustaceans are believed to represent the ancestral arthropods from which insects originated [4]. The evolutionary relationship between these two groups is evident from the common growth strategy of insects and crustaceans that involves molting so that growth can occur. Molting is governed by periodic increases in the levels of $20 \mathrm{E}$ that elicit the programs that coordinate the developmental and metamorphic transitions [5]. Although a great deal of evidence reveals that crustaceans, like insects, synthesize $20 \mathrm{E}$ from cholesterol [6], the molecular details of steroidogenesis in crustaceans remain conjectural. In insects, steroidogenic CYPs are products of the Halloween genes phantom (phm: CYP306A1), disembodied (dib: CYP302A1), shadow (sad: CYP315A1) and shade (shd: CYP314A1) and are responsible for the last four hydroxylations in the pathway leading to 20E [7-14] that is biochemically similar to one that yields $20 \mathrm{E}$ in crustaceans [6] (Fig. 1). In Drosophila melanogaster, mutations in these genes disrupt 20E production and cause the arrest of embryonic development and death. spook (spo: Cyp307a1) is another member of this CYP group which when mutated results in low $20 \mathrm{E}$ mutants $[15,16]$ and is believed to mediate a yet uncharacterized step (the Black Box) in the biosynthesis of 20 E preceding those of Phm, Dib, Sad and Shd. In contrast to $p h m, d i b$, sad and shd for which each insect genome carries one ortholog, several paralogs of spo-like (CYP307) genes have been formed by duplications, which in turn have evolved lineage-specific complements of these genes $[2,16,17]$. For example, Drosophila has two spo-like genes, spo and spookier (spok: Cyp307a2) [16]. These two genes are close paralogs that are believed to mediate the same enzymatic reaction, although at different stages of development.

Although one would expect that orthologs of the insect Halloween genes are present in crustaceans, there is no molecular evidence for the existence of these genes in crustaceans. We have tried for several years to probe hexapod crustaceans for Halloween gene orthologs under various hormonal regimens using degenerate primers based on the Drosophila and Bombyx genes, but we have been unsuccessful (K.F. Rewitz, J.T. Warren, E. Chang and L.I. Gilbert). The development of the genome data base of the more primitive crustacean, Daphnia pulex, allowed us to survey this genome and conduct phylogenetic analyses that suggest strongly that orthologs of spo, phm, dib, sad and shd do exist in Daphnia and thus, in a crustacean i.e. the genes appeared in arthropods before the radiation of insects.

\section{Results and Discussion}

By searching the Daphnia data bases $[18,19]$ we obtained candidate sequences for orthologs of the insect Halloween genes in Daphnia. Single orthologs of phm, dib, sad and shd were retrieved and only one sequence exhibited significant similarity to the spo-like genes in the CYP307 family. We also searched the genomes of non-arthropod invertebrates including the cnidarian Nematostella vectensis, the nematodes C. elegans and Brugia malayi, the annelid Capitella capitata, the mollusk Lottia gigantea, the echinoderm Strongylocentrotus purpuratus $[19,20]$ for Halloween gene orthologs using TBLASTN. Analyses of these invertebrate genomes did not result in any significant hits indicating the absence of Halloween orthologs and neither have we been able to identify orthologs of these genes in any of several vertebrate species.

The genes obtained from Daphnia encode approximately 500 amino acid open reading frames (ORFs) which is typically for proteins belonging to the CYP family [21]. Alignment of Daphnia sequences with orthologs of the insect Halloween genes shows that the genes, in addition to being conserved in areas that comprise canonical structural CYP motifs, exhibit considerable conservation in regions that are believed to determine substrate specificity (Additional file 1). This indicates that the genes are functionally conserved in Daphnia as they are in insects [2]. The overall amino acid identity between deduced orthologous proteins from eight insects belonging to four different orders and the Daphnia orthologs ranges from an average of $55.2 \% \pm 6.8 \mathrm{SD}$ (standard deviation) for Spolike proteins to somewhat lower values for Sad proteins $(38.7 \% \pm 8.8 \mathrm{SD})$. Thus, spo-like genes are the most highly conserved of these genes and this appears to be true for Daphnia like it is for insects. Conservation of these genes from insect to crustaceans, which separated $\sim 400$ millions years ago [4], shows that selection has preserved the genes because of their function. The reason that spo-like genes are more conserved than the other arthropod steroidogenic CYP enzymes is not known, although it may be related to the possibility that Spo acts in the rate-limiting Black Box reaction(s) $[5,22]$. If Spo is involved in control of the flux through the pathway it may have been a particular target for selection because mutations altering its enzyme activity would have had increased consequences compared to mutations altering the activity of enzymes that are less rate-limiting. A phylogenetic analysis was performed using the Daphnia sequences.

\section{Phylogenetic analysis of Daphnia candidate orthologs}

A phylogenetic tree was constructed with Daphnia sequences retrieved from the BLAST searches and orthologs of the insect Halloween genes from Drosophila, red flour beetle Tribolium castaneum and honey bee Apis mellifera, which represent three different orders (Diptera, 

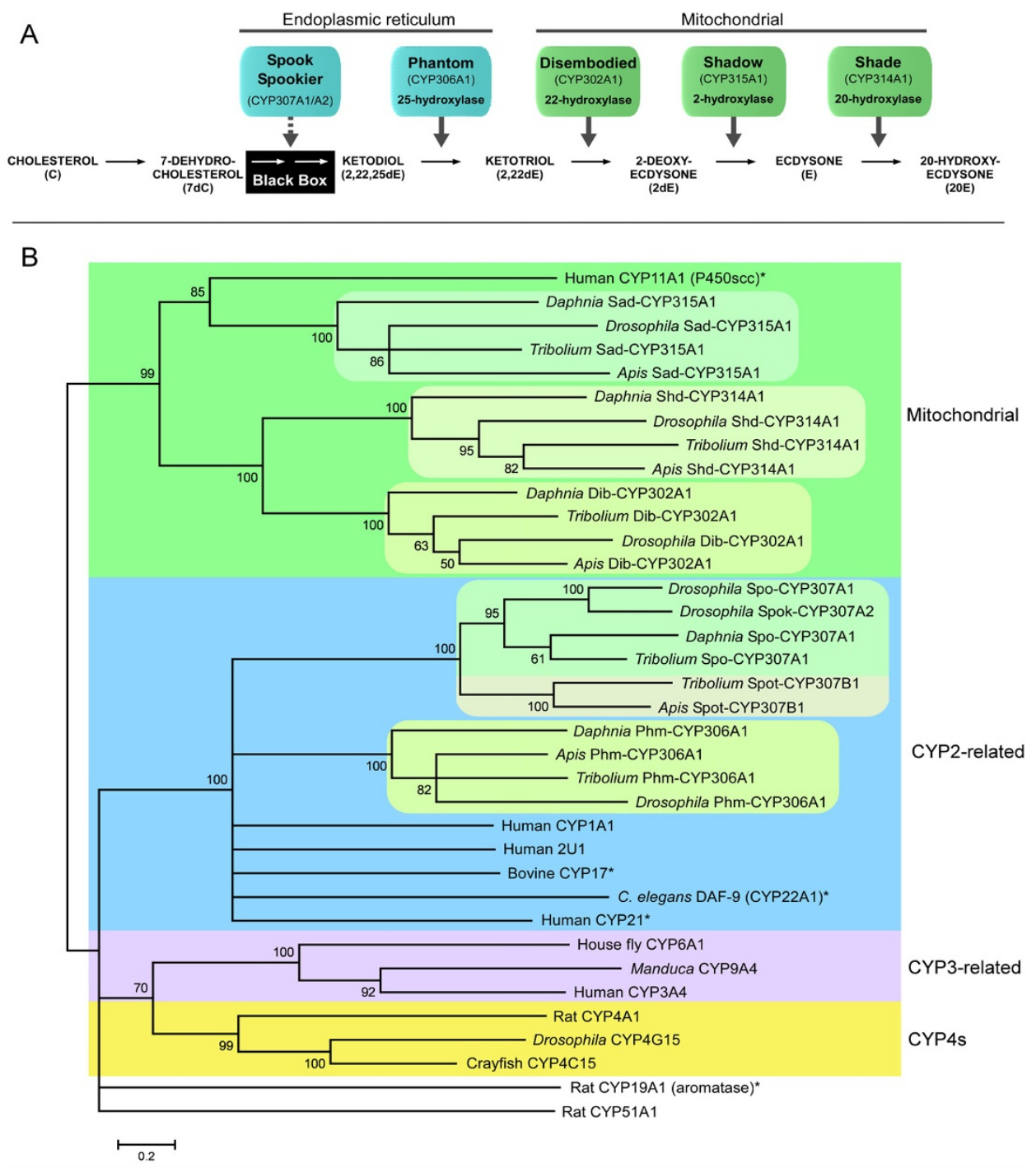

Figure I

Scheme of 20-hydroxyecdysone (20E) biosynthesis and a phylogenetic tree including Daphnia Halloween orthologs. A) Biosynthetic scheme showing the steroidogenic CYP enzymes encoded by genes in the Halloween family mediating steps in the conversion of cholesterol to $20 \mathrm{E}$ and the subcellular distribution of these enzymes. The Black Box denotes an uncharacterized series of oxidative modifications converting 7-dehydrocholesterol (7dC) to the first ecdysteroid-like molecule, namely the ketodiol (2,22,25-trideoxyecdysone: $2,22,25-\mathrm{dE})$ [I]. Dashed arrow indicates that there is no direct evidence for the catalytic function of Spook (Spo) and Spookier (Spok), but several pieces of evidence point to a function of these enzymes in the Black Box [16]. 2,22-dideoxyecdysone (2,22-dE). B) Maximum Likelihood phylogenetic tree showing relationships of the Daphnia sequences with orthologs of the insect steroidogenic CYP products of the Halloween genes Spo, Spok, Spookiest (Spot), Phantom (Phm), Disembodied (Dib), Shadow (Sad), Shade (Shd). Other selected vertebrate and C. elegans steroidogenic and non-steroidogenic CYPs are included to infer relationship to major classes of CYPs. Members of major metazoan CYP classes are represented in this analysis: the mitochondrial, CYP2-related, CYP3-related, CYP4s, CYPI9 and CYP5I. Numbers indicate support values obtained by bootstrapping 100 replicates and branches under the threshold value of 50 are shown as polytomies. Human CYP3A4 (AAI0I632), Rat CYP4AI (NP 78703I), Crayfish CYP4CI5 (AAF09264), Drosophila CYP4GI5 (AAF76522) House fly CYP6AI (AAA29293), Manduca CYP9A4 (AAD5I036), Human CYPI IAI - P450scc cholesterol side chain cleavage enzyme (AAH32329), Bovine CYPI7AI - steroid I $7 \alpha$-hydroxylase/I7,20 lyase (P05I85), Rat CYPI9AI - aromatase converting androgens to estrogens (P22443), Human CYP2I - steroid 2I-hydroxylase (AAB59440), Human CYPIAI (AAH23019), Human CYP2UI (NP_898898), Rat CYP5I - sterol I4 $\alpha$-demethylase (Q64654), and C. elegans DAF-9 (CYP22AI) - produces the steroid ligand for the DAF-I 2 nuclear receptor (AAL65I32) [35]. * Shows CYP enzymes from animals, other than arthropods, that are involved in steroidogenesis. 
Coleoptera and Hymenoptera, respectively) of holometabolous insects. The Daphnia gene products separate with the insect orthologs of Spo/Spok, Phm, Dib, Sad and Shd in this phylogenetic tree with high bootstrap support (Fig. 1). In examining the genome of Daphnia for orthologs of these genes, we noticed no closely related paralogs to the obtained candidate orthologs. This supports the orthology of the genes and indicates that the genes became functionally specialized before the split between crustaceans and insects and have been under heavy selection pressure ever since. Only one spo-like gene was obtained and this gene is phylogenetically a CYP307A subfamily gene i.e. most closely related to $s p o / s p o k$. No ortholog of spookiest (spot: CYP307B1) was found indicating that this gene does not exist in Daphnia, which implies that it is insect-specific i.e. originating from a duplication occurring after insects arose from crustaceans [4] or that it was lost in Daphnia as it has been in some insects e.g. lepidopterans and Drosophila species [2].

In the phylogenetic analysis, sequences of steroidogenic CYPs from vertebrates and C. elegans were included to probe ancestral relationships and the origin of CYPs involved in steroid biogenesis (Fig. 1). Figure 1 includes major groups of metazoans CYPs i.e. mitochondrial, CYP2-related, CYP3-related, CYP4-related, CYP19s and CYP51s. The steroidogenic CYPs of insects and their Daphnia orthologs are evolutionarily related to vertebrate and C. elegans steroidogenic CYPs since they cluster in two major groups, those related to CYP2 enzymes (Spo, Phm, CYP17, CYP21 and DAF-9) and those that are mitochondrial (Dib, Sad, Shd and CYP11A1). Therefore, it is likely that different steroidogenic CYP enzymes are derived from common ancestors and were recruited for steroid biosynthesis prior to the protostome-deuterostome split. Evolution of these ancestral CYPs in C. elegans, arthropods and vertebrates, by gene duplication and divergence, likely shaped the biosynthetic pathways yielding the different groups of steroids in these animals.

\section{Conservation of gene structure}

Comparison of intron positions of the aligned Daphnia protein sequences and the insect orthologs of the Halloween genes shows that many introns are conserved (Fig. 2). Each Daphnia ortholog contains conserved intron/exon organization typical of their respective insect orthologs. The conserved gene structure suggests that the Daphnia genes have a common evolutionary origin with the insect Halloween genes and support the inferred phylogenetic relationship suggesting orthology (Fig. 1). In phm, five introns are conserved among Daphnia and insects and only two introns are unique to the Daphnia gene. Introns that have no counterparts can be explained by intron gain or alternatively these introns have been lost. Interestingly, Daphnia spo has two introns whereas insect spo genes have only one. The position and phase (the nucleotide position in a codon) of introns in the Daphnia spo gene are equivalent to those conserved in insect spot genes. However, the Daphnia sequence is the closest ortholog of the insect spo/ spok genes phylogenetically, and only shares one of these conserved introns. A likely evolutionary scenario is that the ancestral gene was a spo gene containing both introns (Fig. 3). In the insect lineage, after the split from crustaceans, duplication of this gene resulted in two copies that diverged into spo and spot paralogs after which one intron was lost in the insect spo genes.

Although several introns are conserved among the mitochondrial dib, sad and shd, introns that are unique to each group of orthologs (e.g. the first phase 1 intron in $d i b$ and shd) indicate an orthologous relationship to the respective groups. The conserved gene structure of these genes suggests that they evolved from a common ancestor of mitochondrial CYPs by duplications and diverged into the steroidogenic enzymes that are functionally specialized to carry out the last three steps in which C22, C2 and C20 hydroxylations form 20E. This implies, at least in part, that the biosynthetic pathway evolved step-wise by duplication and functional divergence of the biosynthetic enzymes. In vertebrates, functional divergence and specialization of steroidogenic CYPs are known from studies of the CYP11 paralogs. CYP11A1 is the enzyme that mediates the removal of the side chain of cholesterol [23]. CYP11B1 and CYP11B2 are paralogs, from a recent duplication, that became functionally specialized as the steroid $11 \beta$-hydroxylase and the aldosterone synthase, respectively [24]. dib, sad and shd also exhibit conservation of gene structure when compared to vertebrate mitochondrial CYPs involved in steroidogenesis [2].

\section{Microsynteny supports orthology}

The conserved arrangement of genes on the chromosome of different species (microsynteny) can be used to infer phylogenetic relationships that support the orthology of genes $[2,17]$. Analysis of local genome structures surrounding the Halloween genes shows little conservation of microsynteny between insects and crustaceans. This is likely due to the relatively great evolutionary time separating insects from crustaceans. However, remnants showing microsynteny can be found in at least two cases. CYP18A1 is a paralog of phm which is found in Drosophila and most other insects [25]. In Drosophila and Apis, phm and CYP18A1 are arranged tail-to-tail and adjacent to these genes are CG6696 and fused (Fig. 2). In Daphnia, the microsyntenic relationship of phm and CYP18A1 is conserved, although CG6696 and fused appear to have been rearranged to different chromosomal locations.

Comparison of gene organization in a region of approximately $100 \mathrm{~kb}$ surrounding the $s p o(k)$ locus reveals that 
A
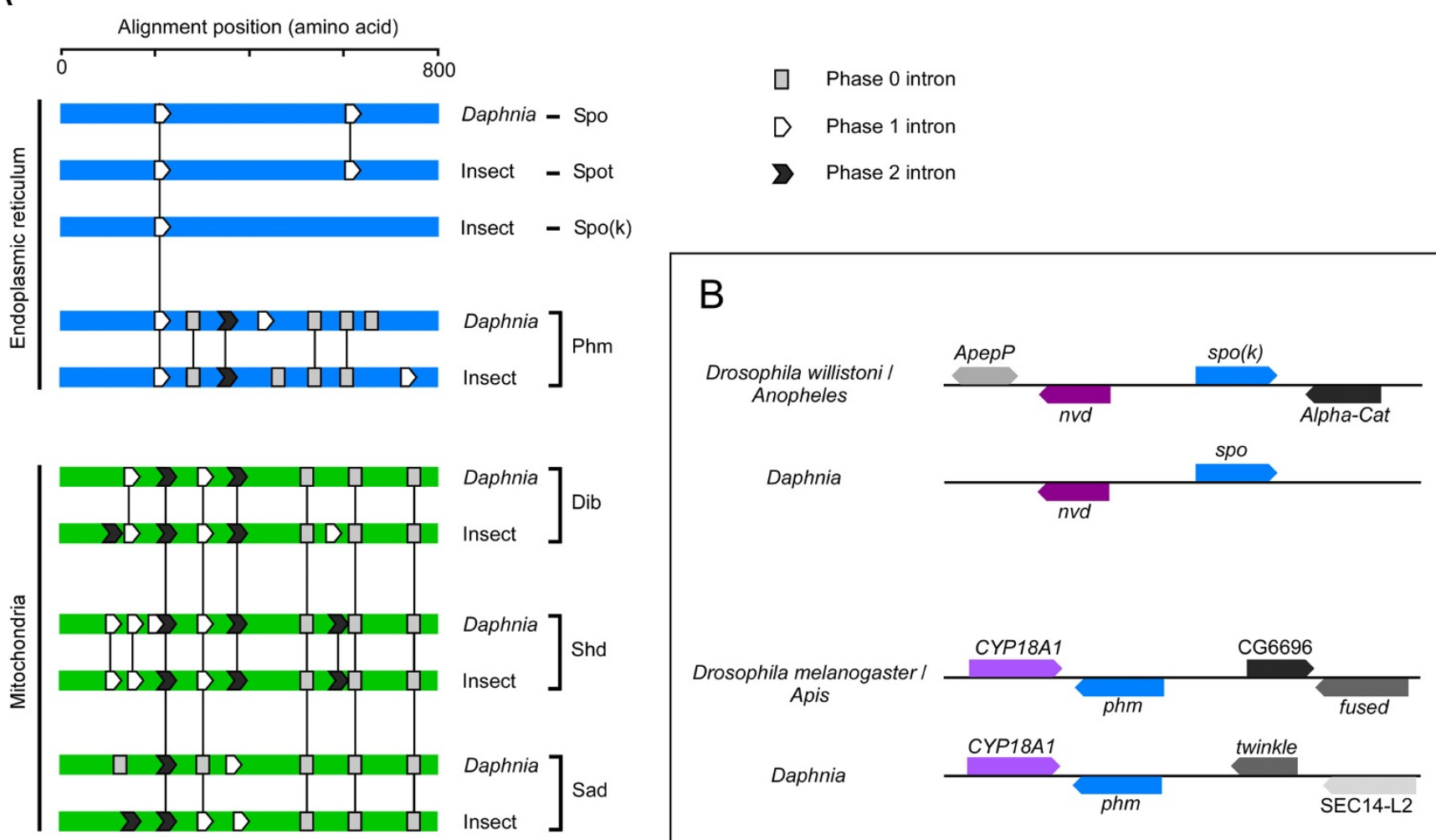

Figure 2

Intron/exon structure and microsynteny of Daphnia and insect Halloween genes. A) Introns are mapped on the aligned protein sequences of Spo (CYP307AI), Spot (CYP307BI), Spok (CYP307A2), Phm (CYP306AI), Dib (CYP302AI), Sad (CYP3I5AI), Shd (CYP3I4AI). Introns located at the same position and in the phase (the nucleotide position of the intron within a codon: phase 0 between codons, phase I after the first base and phase 2 after the second base), on the aligned proteins, are shown as conserved by connecting vertical lines. Insect introns represent introns found in species of insects previously described [2], except Tribolium shd which exhibits unique introns that are not shown. B) Preserved microsynteny in local genome regions surrounding $\mathrm{phm}$ and spo(k). The arrangement of phm and its paralog CYPI8AI is conserved in insects and Daphnia and so is the microsyntenic relationship of spo (spok in Drosophila) and neverland (nvd). Nvd is believed to be involved in the conversion of cholesterol to 7-dehydrocholesterol $(7 \mathrm{dC})$, the step preceding the Black Box in which Spo/Spok may function [16,26]. The transcriptional direction of the genes is shown by arrowheads. The double arrowhead indicates that ApepP is in opposite orientations in Drosophila and Anopheles.

spo is adjacent to neverland (nvd) [26] in Daphnia and the mosquito Anopheles gambiae. Analysis of the genome region of spo-like genes in species of Drosophila (available at FlyBase [27]) shows that in Drosophila pseudoobscura, Drosophila willistoni and Drosophila mojavensis nvd is located within $60 \mathrm{~kb}$ of spok. This is in agreement with the view that spok is the ancestral gene in Drosophilidae. Intriguingly, $\mathrm{Nvd}$ is a conserved Rieske-like oxygenase believed to be involved in the conversion of cholesterol to 7-dehydrocholesterol in insects and C. elegans [26,28]. In insects, this is presumably the first step in the biosynthetic pathway of $20 \mathrm{E}$ from cholesterol via 7-dehydrocholesterol, the latter being the substrate that enters the Black Box, in which Spo/Spok likely participate (although at different developmental stages) to produce the substrate for
Phm, the ketodiol (Fig. 1). Although unequivocal evidence for the exact function of Nvd and Spo/Spok is lacking, experiments utilizing ecdysteroid precursors to rescue mutant phenotypes (RNAi-induced for $n v d$ and spok) suggest that in Drosophila nvd and spo/spok genes function in these two succeeding steps in the biochemical pathway $[16,26]$. However, it must be emphasized that the Black Box may hold more than one reaction and more than one enzyme may be participating in this conversion [5].

\section{Conclusion}

Currently, it is clear that CYPs are involved in steroid biosynthesis in vertebrates and invertebrates. In C. elegans, insects and vertebrates, the majority of steroidogenic CYPs are related to one of two groups, the CYP2 enzymes and 


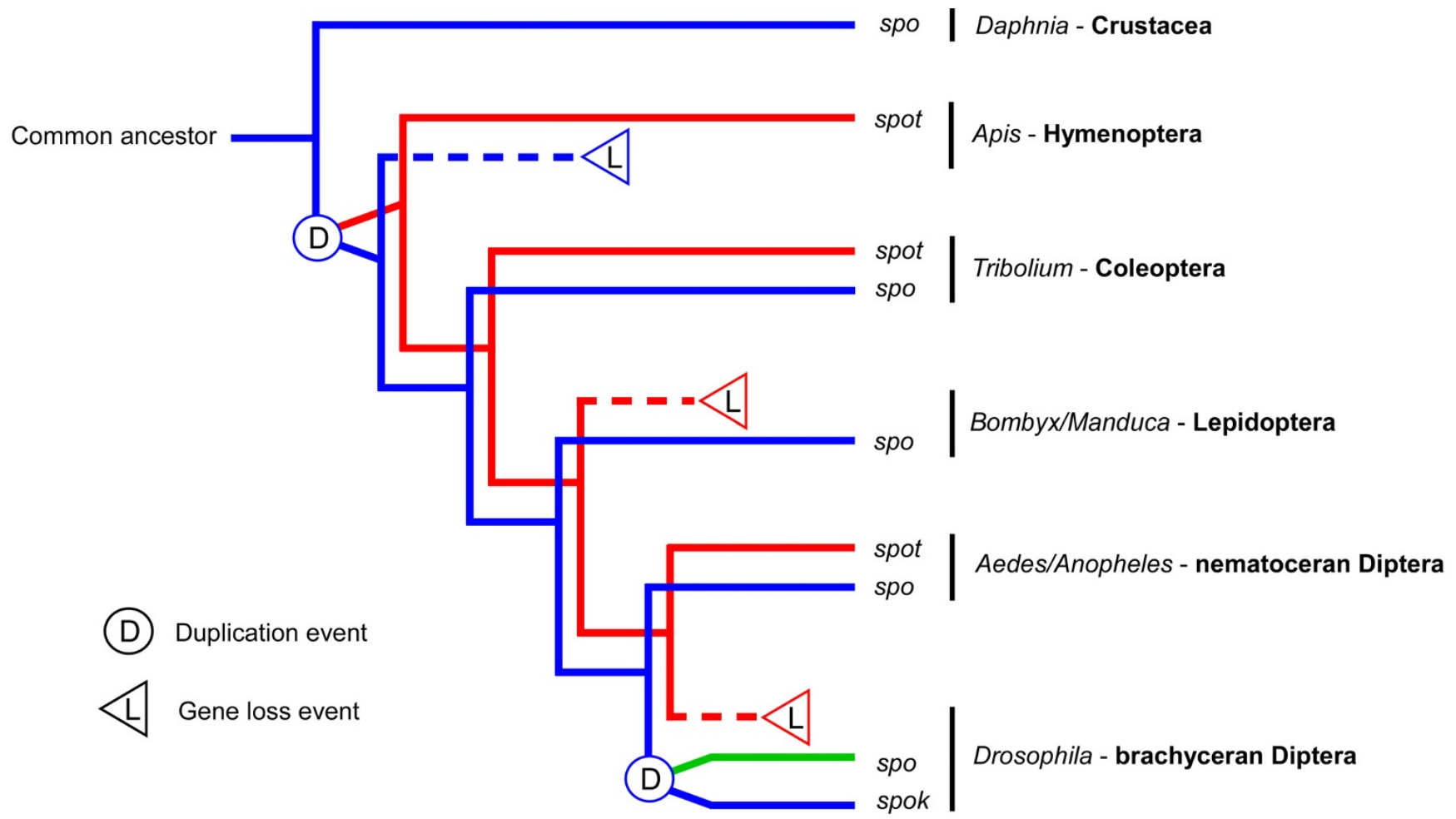

Figure 3

Lineage-specific duplications and losses of spo-like genes in Daphnia and insects. An evolutionary scenario based on the observed distribution of spo-like (CYP307) genes in arthropods. Since insects are believed to have evolved from crustaceans living in freshwater environments [4] and the only spo-like gene observed in Daphnia belongs to the CYP307A subfamily, the ancestral arthropod spo gene was likely a CYP307A gene. An early duplication, which probably occurred after insects diverged from crustaceans, gave rise to spo (CYP307AI) and spot (CYP307BI). In Drosophila, the ancestral spo-like gene, referred to as spok (Сyp307a2), underwent a second round of duplication in which an intronless retrogene arose. In Drosophila this gene is referred to as spo. Note that another round of gene duplication, which occurred in the line of Drosophila evolution leading to the subgenus Drosophila [17], is not shown.

the mitochondrial CYPs. It is therefore likely that these steroidogenic CYPs evolved from common ancestors which had the capacity to modify cholesterol before the split of these animals. This implies that the distinct biosynthetic pathways in these metazoans, which convert cholesterol to different types of steroids, likely result from lineage-specific evolution of ancestral steroidogenic CYPs. The results suggest strongly that steroidogenic CYP enzymes mediating the biosynthesis of $20 \mathrm{E}$ are present in crustaceans and are relegated to the Arthropoda. Therefore, these enzymes probably became functionally specialized in the line leading to arthropods. Although functional characterization of the gene products, such as that done for the insect Halloween genes [1,29], is required to obtain unequivocal evidence of their role in 20 E production in Daphnia, the present data provide the first evidence for steroidogenic CYP genes in crustaceans as well as the groundwork for future functional genomic analyses in the field of crustacean endocrinology.

\section{Methods}

\section{Data base search}

The Daphnia pulex genome sequence (v1.0) made available by the Daphnia Genomics Consortium and the DOE Joint Genome Institute [19] was mined for orthologs of the insect Halloween genes using TBLASTN searches. Apis and Tribolium sequences, previously described in Rewitz et al. [2], were used as queries to identify homologous sequences in the Daphnia data base. Sequences of insect Halloween genes, previously described in Rewitz et al. [2], were gleaned from FlyBase [27] and Rene Feyereisen's website for insect CYPs [30]. C. elegans and vertebrate CYP protein sequences were acquired from NCBI [20].

\section{Phylogenetic tree construction, intron positions and microsynteny analysis}

Predicted Daphnia orthologs of the insect Halloween genes were analyzed using a phylogenetic tree with insect Halloween genes from insects belonging to three different 
orders, Apis (Hymenoptera), Tribolium (Coleoptera) and Drosophila (Diptera). To infer phylogenetic relationships of these steroidogenic CYPs with other members of this multigene family, steroidogenic and non-steroidogenic CYPs representing some of the major groups of metazoan CYPs were included in this analysis i.e. mitochondrial, CYP2-related, CYP3-related and CYP4s, CYP19 and CYP51. Deduced Daphnia protein sequences used in the alignment can be found in Additional file 2. A multiple alignment of the protein sequences was constructed with ClustalX (1.83) [31] and manually edited using SeaView [32]. A phylogenetic tree was constructed from this alignment using the Maximum Likelihood method under the Jones-Taylor-Thornton (JTT) substitution model using PHYML (v2.4.5) [33]. Support values were obtained by bootstrapping 100 replicates. Branches with bootstrap support below 50 were collapsed to form polytomies.

Gene structure, that is, intron position and phase (the nucleotide position of the intron within a codon: phase 0 between codons, phase 1 after the first base and phase 2 after the second base) were predicted from manual annotation of the Daphnia genes with support from expressed sequence tags (ESTs) data and by homology to insect Halloween genes for which gene structures are known. Intron position and phase were mapped onto a multiple alignment to investigate conservation of intron/exon structure in relation to phylogeny.

Additional support for orthology of Daphnia and insect genes was sought by analyses of microsynteny (conserved order of genes). Genome regions surrounding the candidate Halloween orthologs of Daphnia and several insects were inspected for ORFs encoding conserved genes. Putative gene orthologs exhibiting conserved arrangement in relation to the Halloween orthologs were tested for orthology by reciprocal best hit Blast searches in which orthologs of each species were BLAST searched against the genome of the other species to look for best hits (putative orthologs).

\section{Abbreviations}

20E (20-hydroxyecdysone); Dib (DisembodiedCYP302A1); Nvd (Neverland); ORF (open reading frame); Phm (Phantom-CYP306A1); Sad (ShadowCYP315A1); Shd (Shade-CYP314A1); Spo (SpookCYP307A1); Spok (Spookier-CYP307A2); Spot (Spookiest-CYP307B1); Spo-like (CYP307 family)

\section{Authors' contributions}

KFR conducted the actual phylogenetic and other analyses in this paper and wrote the first draft. LIG came up with the idea of identifying the Halloween genes in a crustacean, gained access to the Daphnia data base, recommended that KFR do the search analyses and helped write the final draft of the manuscript. All authors read and approved the final manuscript.

\section{Additional material}

\section{Additional File 1}

Putative substrate recognition sites (SRSs).

Click here for file

[http://www.biomedcentral.com/content/supplementary/1471-

2148-8-60-S1.pdf]

\section{Additional File 2}

Deduced protein sequences of Daphnia pulex Spo-CYP307A1, PhmCYP306A1, Dib-CYP302A1, Sad-CYP315A1 and Shd-CYP314A1. Click here for file

[http://www.biomedcentral.com/content/supplementary/14712148-8-60-S2.pdf]

\section{Acknowledgements}

We offer special thanks to John Colbourne and Joseph Shaw for their help in gaining access to the Daphnia data base and Michael B. O'Connor and James T. Warren for their continued collaboration on the Halloween gene project. The Daphnia sequence data were produced by the US Department of Energy Joint Genome Institute [19] in collaboration with the Daphnia Genomics Consortium [34]. Our work benefits from, and contributes to, the Daphnia Genomics Consortium. The present work was supported by grant 0516623 from the National Science Foundation.

\section{References}

I. Gilbert LI, Warren JT: A molecular genetic approach to the biosynthesis of the insect steroid molting hormone. Vitam Horm 2005, 73:3I-57.

2. Rewitz KF, O'Connor MB, Gilbert LI: Molecular evolution of the insect Halloween family of cytochrome P450s: phylogeny, gene organization and functional conservation. Insect Biochem Mol Biol 2007, 37:74I-753.

3. Lafont R, Mathieu M: Steroids in aquatic invertebrates. Ecotoxicology 2007, I 6:109-130.

4. Glenner H, Thomsen PF, Hebsgaard MB, Sørensen MV, Willerslev E: The origin of insects. Science 2006, 31 4: I 883-1884.

5. Gilbert LI, Rybczynski R, Warren JT: Control and biochemical nature of the ecdysteroidogenic pathway. Ann Rev Entomol 2002, 47:883-916.

6. Lachaise F, Le Roux A, Hubert M, Lafont R: The molting gland of crustaceans: localization, activity, and endocrine control. J Crustacean Biol 1993, 13:198-234.

7. Chavez VM, Marques G, Delbecque JP, Kobayashi K, Hollingsworth M, Burr J, Natzle JE, O'Connor MB: The Drosophila disembodied gene controls late embryonic morphogenesis and codes for a cytochrome P450 that regulates embryonic ecdysone levels. Development 2000, I27(19):4II5-4I26.

8. Petryk A, Warren JT, Marques G, Jarcho MP, Gilbert LI, Parvy J-P, Dauphin-Villemant C, O'Connor MB: Shade is the Drosophila P450 enzyme that mediates the hydroxylation of ecdysone to the steroid insect molting hormone 20-hydroxyecdysone. Proc Natl Acad Sci USA 2003, 100:13773-13778.

9. Niwa R, Matsuda T, Yoshiyama T, Namiki T, Mita K, Fujimoto $Y$, Kataoka H: CYP306AI, a cytochrome P450 enzyme is essential for ecdysteroid biosynthesis in the prothoracic glands of Bombyx and Drosophila. J Biol Chem 2004, 279:35942-35949.

10. Niwa R, Sakudoh T, Namiki T, Saida K, Fujimoto Y, Kataoka H: The ecdysteroidogenic CYP CYP302A I/disembodied from the silkworm, Bombyx mori, is transcriptionally regulated by prothoracicotropic hormone. Insect Mol Biol 2005, I 4:563-57I. 
II. Rewitz KF, Rybczynski R, Warren JT, Gilbert LI: Identification, characterization and developmental expression of Halloween genes encoding P450 enzymes mediating ecdysone biosynthesis in the tobacco hornworm, Manduca sexta. Insect Biochem Mol Biol 2006, 36: 188-199.

12. Rewitz KF, Rybczynski R, Warren JT, Gilbert LI: Developmental expression of Manduca shade, the P450 mediating the final step in molting hormone synthesis. Mol Cell Endocrinol 2006 247: 166-I74.

13. Warren JT, Petryk A, Marques G, Jarcho M, Parvy J-P, Dauphin-Villemaont C, O'Connor MB, Gilbert LI: Molecular and biochemical characterization of two P450 enzymes in the ecdysteroidogenic pathway of Drosophila melanogaster. Proc Natl Acad Sci USA 2002, 99: I 1043-II048.

14. Warren JT, Petryk A, Marques G, Jarcho M, Parvy JP, Shinoda T, Itoyama K, Kobayashi J, Jarcho M, Li Y, O'Connor MB, Dauphin-Villemant C, Gilbert LI: Phantom encodes the 25-hydroxylase of Drosophila melanogaster and Bombyx mori: a P450 enzyme critical in ecdysone biosynthesis. Insect Biochem Mol Biol 2004, 34:991-1010.

15. Namiki T, Niwa R, Sakudoh T, Shirai K, Takeuchi H, Kataoka $H$ : Cytochrome P450 CYP307A I/Spook: a regulator for ecdysone synthesis in insects. Biochem Biophys Res Comm 2005 337:367-374.

16. Ono H, Rewitz KF, Shinoda T, Itoyama K, Petryk A, Rybczynski R, Jarcho M, Warren JT, Marques G, Shimell MJ, Gilbert LI, O'Connor MB: Spook and spookier code for stage-specific components of the biosynthetic pathway in Diptera. Dev Bio 2006 298(2):555-5780. Epub 2006 Jul 29.

17. Sztal T, Chung H, Gramzow L, Daborn PJ, Batterham P, Robin C: Two independent duplications forming the Cyp307a genes in Drosophila. Insect Biochem Mol Biol 2007, 37: 1044-1053.

18. Daphnia genome database [http://wFleaBase.org]

19. DOE Joint Genome Institute [http://jgi.doe.gov/]

20. NCBI [http://ncbi.nlm.nih.gov/]

21. Feyereisen R: Insect cytochrome P450. In Comprehensive Molecular Insect Science Volume 4. Edited by: Gilbert LI, latrou K. Gill S: Elsevier; Oxford; 2005: I-77.

22. Lafont R, Dauphin-Villemant C, Warren JT, Rees H: Ecdysteroid chemistry and biochemistry. In Comprehensive Molecular Insect Science Volume 3. Edited by: Gilbert LI, latrou K. Gill S: Elsevier; Oxford; 2005: 125-195.

23. Lisurek M, Bernhardt R: Modulation of aldosterone and cortisol synthesis on the molecular level. Mol Cell Endocrinol 2004, 215:149-159.

24. Bülow HE, Bernhardt R: Analyses of the CYPIIB gene family in the guinea pig suggest the existence of a primordial CYPI IB gene with aldosterone synthase activity. Eur J Biochem 2002, 269:3838-3846

25. Claudianos C, Ranson H, Johnson RM, Biswas S, Schuler MA, Berenbaum MR, Feyereisen R, Oakeshott JG: A deficit of detoxification enzymes: pesticide sensitivity and environmental response in the honeybee. Insect Mol Biol 2006, 15:6I5-636.

26. Yoshiyama T, Namiki T, Mita K, Kataoka H, Niwa R: Neverland is an evolutionarily conserved Rieske-domain protein that is essential for ecdysone synthesis and insect growth. Development 2006, 133:565-2574.

27. FlyBase [http://flybase.bio.indiana.edu/]

28. Rottiers V, Motola DL, Gerisch B, Cummins CL, Nishiwaki K, Mangelsdorf DJ, Antebil A: Hormonal control of C. elegans Dauer formation and life span by a Rieske-like oxygenase. Dev Cell 2006, 10:473-482.

29. Rewitz KF, Rybczynski R, Warren JT, Gilbert LI: The Halloween genes code for cytochrome $\mathbf{P 4 5 0}$ enzymes mediating synthesis of the insect moulting hormone. Biochem Soc Trans 2006, 34: $1256-1260$

30. Rene Feyereisen's website for insect CYPs [http://p450.anti bes.inra.fr/]

31. Thompson JD, Gibson TJ, Plewniak F, Jeanmougin F, Higgins D: The CLUSTALX windows interface: flexible strategies for multiple sequence alignment aided by quality analysis tools. Nucleic Acids Res 1997, 25:4876-4882.

32. Galtier N, Gouy M, Gautier C: SEAVIEW and PHYLO_WIN two graphic tools for sequence alignment and molecular phylogeny. Comput Appl Biosci 1996, I 2:543-548.
33. Guindon S, Gascuel O: A simple, fast, and accurate algorithm to estimate large phylogenies by maximum likelihood. Syst Biol 2003, 52:696-704.

34. Daphnia Genomics Consortium [http://daphnia.cgb.indiana.edu/ ]

35. Motola DL, Cummins CL, Rottiers V, Sharma KK, Li T, Li Y, SuinoPowell K, Xu HE, Auchus RJ, Antebi A, MangelsdorfI DJ: Identification of ligands for DAF- 2 that govern Dauer formation and reproduction in C. elegans. Cell 2006, I24:|209-|223.
Publish with Bio Med Central and every scientist can read your work free of charge

"BioMed Central will be the most significant development for disseminating the results of biomedical research in our lifetime. "

Sir Paul Nurse, Cancer Research UK

Your research papers will be:

- available free of charge to the entire biomedical community

- peer reviewed and published immediately upon acceptance

- cited in PubMed and archived on PubMed Central

- yours - you keep the copyright 